\title{
Comparative Study of Different Column Types for the Separation of Polar Basic Hallucinogenic Alkaloids
}

\author{
Somandla Ncube ${ }^{a}$, Anna Poliwoda ${ }^{b}$, Ewa Cukrowska ${ }^{a}$, Piotr Wieczorek ${ }^{b}$ and Luke Chimuka ${ }^{a, k}$ \\ ${ }^{a}$ Molecular Sciences Institute, School of Chemistry, University of the Witwatersrand, Johannesburg, 2050, South Africa. \\ ${ }^{b}$ Faculty of Chemistry, Opole University, Pl. Kopernika 11, 45-040 Opole, Poland.
}

Received 13 April 2016, revised 30 June 2016, accepted 19 July 2016.

\begin{abstract}
The number of hallucinogenic compounds that have been separated simultaneously by liquid chromatography is limited. This research aimed to identify a column(s) that can allow for separation of several hallucinogens. The extent of separation of seven polar hallucinogenic tryptamine and phenethylamine derived alkaloids containing a basic $\mathbf{N}$ atom that becomes protonated at low $\mathrm{pH}$ values were investigated on five reverse-based columns and one hydrophilic interaction liquid chromatography (HILIC) column. An RP-phenyl and a negatively charged fused core HILIC were identified and recommended as effective columns in this regard. This research is the first to introduce a HILIC column in separation of hallucinogenic alkaloids and to simultaneously study the separation of the two principal psychoactive agents, muscimol and psilocin. The results of this study showed that better separation in HILIC columns can be achieved if an aprotic diluent of relatively lower polarity is used to introduce analytes into a mobile phase of higher polarity. Tetrahydrofuran was recommended where acetonitrile is used as the organic component of the mobile phase.
\end{abstract}

KEYWORDS

Hallucinogen, polar compounds, psilocin, muscimol, HILIC, tetrahydrofuran.

\section{Introduction}

Hallucinogens are pharmacologically psychoactive agents that have mind-altering effects and result in non-ordinary psychological experiences. ${ }^{1}$ A hallucinogen can be psychedelic, dissociative or deliriant depending on its subjective and behavioural effects. Common are the psychedelics, which are structurally classified as tryptamine or phenethylamine derivatives. Mushrooms in the Amanita and Psilocybe genera are known to have hallucinogenic properties due to the presence of ibotenic acid and psilocybin as well as their main respective metabolites, muscimol and psilocin, which are considered the principal psychoactive agents. ${ }^{2-4}$ The toxicity and psychological consequences of the main psychoactive agents are well documented. ${ }^{1,3-11}$

Even though research in hallucinomics is limited, some articles have reported chromatographic quantitation methods with all liquid chromatography using reverse phase columns. ${ }^{4,12-32}$ Our research observed that there has never been an attempt to simultaneously quantify muscimol and psilocin despite being identified as the psycho-excitatory agents responsible for the hallucinogenic effects of mushrooms. Studies in which either of the two alkaloids is separated or quantified simultaneously with other compounds, whether hallucinogenic or non-hallucinogenic, have included derivatization or ion-pairing. ${ }^{4,14,21,23,31,32}$ Pichini et al..$^{33}$ demonstrated the separating ability of ultra-high-pressure liquid chromatography tandem mass spectrometry (UHPLCMS/MS) where a C18 column was used to separate psilocin from four other hallucinogenic alkaloids. The absence of publications on muscimol and psilocin, and the limited number of articles on simultaneous study of hallucinogens, might be an indication that the reverse phase alkyl columns are not the best in separating polar basic compounds as represented by the hallucinogenic alkaloids, a situation concurred by other researchers. ${ }^{23,34,35}$ ${ }^{*}$ To whom correspondence should be addressed. E-mail: luke.chimuka @ wits.ac.za
Almost all hallucinogens are alkaloids and contain an aromaticalkyl-N moiety that is basic at the $\mathrm{N}$ atom, except salvinorin $\mathrm{A}$ and tetrahydrocannabinol. The pentafluorophenylpropyl (PFP) stationary phase has been used successfully in separating some phenethylamine alkaloids. ${ }^{35-37}$ It has also been used on a combined mixture of eight tryptamine and phenethylamine derivatives in $A$. rigidula and its dietary extracts with only two analytes co-eluting. ${ }^{34}$ When a $C_{18}$ column was used, five of the eight analytes could not be baseline separated. PFP is itself a reverse phase approach and has shown great separating ability for basic $\mathrm{N}$ based compounds. Brandt and Martins ${ }^{26}$ have replaced particlepacked reverse phase columns with porous monolithic packing in $\mathrm{C}_{18}$ columns in separating $24 \mathrm{~N}$, N-dialkylated tryptamines using liquid chromatography-electrospray ionization-ion trapmass spectrometry/ mass spectrometry.

Even though the two psychoactive agents have a minimal chance of coexisting in mushrooms, an approach that allows for their simultaneous quantification in body fluids of victims or culprits of neuro-excitatory mushroom consumption will help in saving time towards proving prior exposure to a specific mushroom genus. Our research thus aimed to identify column types that can enable a successful separation and quantification of hallucinogenic alkaloids using a liquid chromatographic approach. A successful concurrent study of the hallucinogenic alkaloids can help maximize the number of these alkaloids that can be analyzed in biological samples. The results of our study can also help in affording an informed starting point for the separation of related compounds by liquid chromatography. Muscimol and psilocin and their precursors, tryptophan and tryptamine, were used as model diamine alkaloid compounds. Included was the monoamine phenethylamine and its two derivatives, 3,4-dimethoxyphenethylamine and hordenine. Despite having different chemical structures, these tryptamine 
and phenylalkylamine derivatives all contain a substituted ethylamine group that is attached to a ring and also produce remarkably similar subjective effects in the human body as neurotransmitter agonists. ${ }^{1,2,6,18,20,38,39}$ Only muscimol has a methylamine group connected to a five-membered ring. The alkylamine group in hallucinogens found in mushrooms is connected to a five-membered ring while the Cactus-sourced have this branch connected to a six-membered ring (Fig. 1).

The optimum separation of the seven alkaloids was investigated on six columns. This was achieved by investigating parameters responsible for maximizing separation on two alkyl reverse phase (RP-alkyl) columns, a reverse phase amide (RP-amide), two phenyl based reverse phase columns and a HILIC column.

\section{Experimental}

\subsection{Chemicals}

All compounds except psilocin (PSI) were purchased from Sigma-Aldrich (Johannesburg, South Africa). PSI in powder form was obtained via the Department of Forensic Sciences, Capetown, South Africa. Muscimol (MUS), tryptophan (TRP), tryptamine (TA) and 3,4- dimethoxyphenethylamine (DMPE) were all purchased in powder form while phenethylamine (PEA) and hordenine (HO) were in liquid form. Ammonium acetate, ammonium formate, acetic acid and formic acid were also purchased from Sigma-Aldrich (Johannesburg, South Africa). HPLC-grade acetonitrile $(\mathrm{MeCN})$, methanol $(\mathrm{MeOH})$, acetone and tetrahydrofuran (THF) were obtained from Merck (Pty) Ltd, Johannesburg, South Africa. Deionized water used to prepare standard solutions and the mobile phase was purified using a Milli-Q-RO4 system (Millipore, Bedford, MA, USA). All six columns investigated were purchased from Sigma-Aldrich (Johannesburg, South Africa).

\subsection{Standards}

The injection standards were prepared by dissolving appropriate volumes of the stock solutions into deionized water, $\mathrm{MeOH}, \mathrm{MeCN}$, THF or acetone depending on the parameter and the column being investigated. Stability issues associated with psilocin and muscimol ${ }^{20,29,40}$ and the difficulty in their procurement limited the quantities sufficient enough for this analytical research. Psilocin and muscimol were then injected at 1 and $2 \mu \mathrm{g} \mathrm{mL}^{-1}$ respectively. The other analytes were injected at $4 \mu \mathrm{g} \mathrm{mL}^{-1}$.<smiles>CN(C)CCc1c[nH]c2cccc(O)c12</smiles>

Psilocin $\mathrm{X} \log \mathrm{P} 3=2.1$ pKa $8.5(\mathrm{~N})$ $11.3(\mathrm{OH})$<smiles>NCCc1ccccc1</smiles>

Phenethylamine $\mathrm{X} \log \mathrm{P} 3=1.4$ pKa $9.8(\mathrm{~N})$<smiles>NCCc1c[nH]c2ccccc12</smiles>
$\mathrm{X} \log \mathrm{P} 3=1.6$ pKa $10.2(\mathrm{~N})$

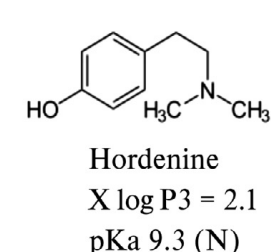

$10.7(\mathrm{OH})$ Tryptamine

\subsection{Instrumentation}

A Bishoff LC-CaDI 22-14 system (Leonberg, Germany) with a Lambda 1010 UV detector set at $280 \mathrm{~nm}$ was used for quantification. Elution was done by isocratic means and the analyte injection was done using Rheodyne Series 7725i manual injector with $10 \mu \mathrm{l}$ sample loop. Prior to elution, each column was equilibrated with the mobile phase for at least $15 \mathrm{~min}$. After each HILIC investigation, the column was first washed with a $\mathrm{MeCN}: \mathrm{H}_{2} \mathrm{O}$ mobile phase containing the same organic component composition as the last mobile phase used for elution and then finally with $100 \% \mathrm{MeCN}$ for $10 \mathrm{~min}$.

\subsection{Column and Hallucinogen Properties}

The physicochemical properties of the columns and the compounds used are given in Table 1 and Fig. 1, respectively. $\mathrm{X} \log \mathrm{P} 3$ is an atom based additive strategy for predicting the octanol/water partition coefficient (log P). Developed by Cheng et $a l .{ }^{41}$, it estimates the $\log \mathrm{P}$ value by adding the contributions from individual atoms constituting the compound and some correction factors. The alkyl RP columns are the most used in liquid chromatographic separations of hallucinogenic alkaloids. Active phase-analyte interactions are exclusively hydrophobic. The RP-amide was chosen for the presence of the embedded polar amide group, which is expected to improve retention of polar analytes and also because it has always been viewed as one of the first definite alternative to $C_{18}$ columns for analysis of nonpolar compounds.

The phenyl-based columns were targeted because of the $\tau-\tau$ interactions with the cyclic groups of the target compounds. The negatively charged fused-core silica Ascentis Express HILIC choice was based on studies by Chirita et al. and recommendations by McCalley considering that our model compounds have positively charged $\mathrm{N}$ atoms at low $\mathrm{pH}$ values. ${ }^{42,43}$

\subsection{Optimization of Column Parameters}

The selection of columns suitable for separation of alkaloids was achieved by optimizing each column parameter. The initial conditions for all parameters investigated were based on the principles of retention for each type of column. The mobile phase was always buffered at $\mathrm{pH} 3$ with formate. Under these conditions all our research compounds were positively charged on the basic $\mathrm{N}$ atom, while the $-\mathrm{OH}$ group remained neutral except for TRP whose pKa $(\mathrm{OH})$ value makes it zwitterionic. While the reversed phase principle requires that the analytes be<smiles>NC(Cc1c[nH]c2ccccc12)C(=O)O</smiles>

Tryptophan $\mathrm{X} \log \mathrm{P} 3=1.6$ pKa $9.4(\mathrm{~N})$ $2.4(\mathrm{OH})$<smiles>NCc1cc(O)no1</smiles>

Muscimol

$\mathrm{X} \log \mathrm{P} 3=-1.1$

pKa $4.8(\mathrm{~N})$

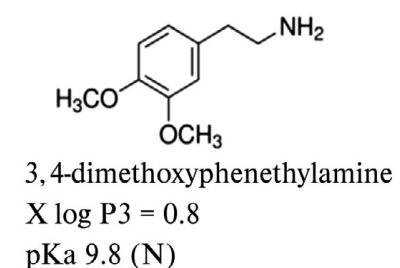

Figure 1 Chemical structures of the model compounds and their relevant physical properties. 
in their neutral state for effective retention, the limiting factor was the pKa values (Fig. 1) that lie outside the $\mathrm{pH}$ 2-8 working range of most columns. All mobile phases in quantitation of hallucinogenic compounds use acetate and formate buffers. ${ }^{4,12-32}$ The analytes are charged in the $\mathrm{pH}$ working range of these buffer systems.

The formate buffering system was prepared only when needed by mixing appropriate quantities to create a specific $\mathrm{pH}$ value buffering system. The reported formate concentrations were the final concentrations in the mobile phase. Because of its low viscosity ${ }^{44-48}, \mathrm{MeCN}$ was used as the organic component of the mobile phase. The chromatographic results presented in this study were for the optimized parameters and for those columns where a separation was observed.

The parameters investigated for alkyl columns included the mobile phase composition and salt concentration of the buffer, flow rate and analyte injection solvent at ambient temperatures. Chirita et al. has suggested the use of HILIC columns for separation of polar analytes. ${ }^{42}$ While the essential parameters in HILIC separation are known ${ }^{42,44,49-51}$, our research further investigated the effect of analyte injection diluents. Water, methanol, acetonitrile, acetone and tetrahydrofuran were investigated as potential analyte diluents. These solvents were chosen based on polarity, eluotropic order and their involvement in other procedures of the research.

\section{Results and Discussion}

\subsection{Alkyl Columns}

The composition of the mobile phase was altered up to Buffer: $\mathrm{MeCN} \mathrm{80:20 \%} \mathrm{(v/v)} 100 \mathrm{mM}$ formate, beyond which it was apparent that the seven compounds could not be separated with most eluting in the column void volume and all detected within

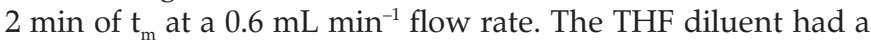
comparably superior retention power even though all analytes eluted before the THF solvent, a situation also observed by Loeser and Drumm. ${ }^{52}$ Solvophilic effects due to high polarity and the charged state of these basic analytes might explain the reduced retention behaviour. ${ }^{53}$

\subsection{Ascentis Hexyl Phenyl Column}

Better retention and separation was observed when the above alkyl column conditions were applied. This was attributed to the presence of the $\pi$-electron phenyl group. The structural position of the benzyl group in TA, in addition to the hydrophobic nature of the ethyl connecting group, might have explained its position in the elution order (Fig. 2).

TRP has a similar positional benzyl group, but TRP is also an amino acid. Both PSI and HO have a phenolic ring and their $X \log$ P3 values are the same, but PSI also has a pyrrole ring joining the phenolic group and the ethyl-amino group. The electron-withdrawing methoxy group in DMPE might explain its retention behaviour compared to PEA. Generally, the three indolealkyltryptamine derivatives (TA, TRP and PSI) could be separated based on type of substituents on the indole ring. This seemed to be the case too with the phenethylamine alkaloids. $\mathrm{HO}$, TRP and DMPE were less retained by the hexyl phenyl column resulting in co-elution.

\subsection{Ascentis Phenyl Column}

Better retention and separation compared to the alkyl and the alkyl phenyl columns was observed. The elution order between TA and PSI was reversed, PEA and DMPE co-eluted while TRP, $\mathrm{HO}$ and DMPE became clearly resolved. Compared to the com- 


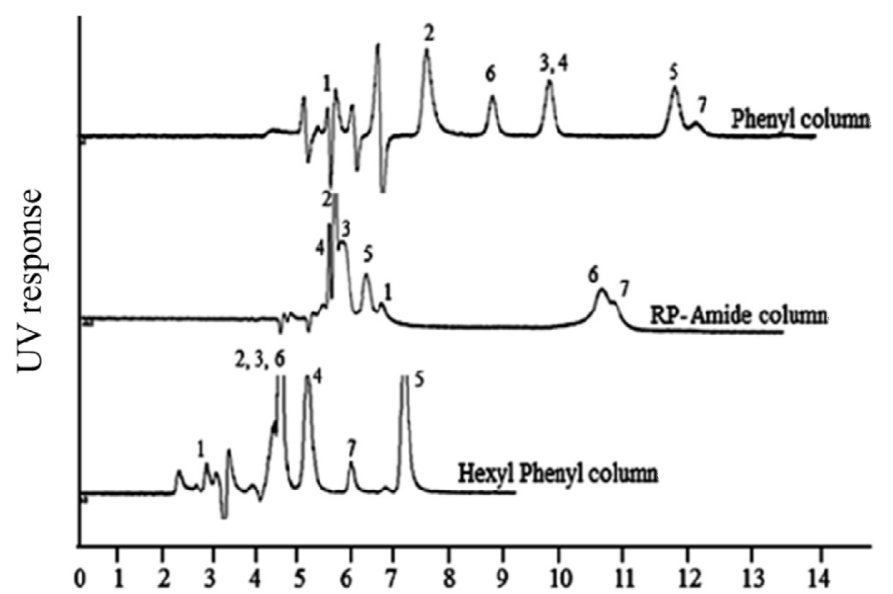

Retention time, $\mathrm{t}_{\mathrm{R}} / \mathrm{min}$

Figure 2 Retention and separation for analytes dissolved in $\mathrm{MeOH}$ on three RP-columns at different optimized conditions. Compounds (descending polarity as given by $\mathrm{X} \log \mathrm{P} 3$ values): (1) MUS; (2) TRP; (3) DMPE; (4) PEA; (5) TA; (6) HO; (7) PSI.

peting effect of the hydrophobic hexyl spacer in hexyl phenyl column, the butyl linking group in the phenyl column allowed for stronger $\pi-\pi$ electron interactions between the phenyl group and the cyclic groups of the analyte. The shorter linking group in the phenyl column also allows for some kind of hydrophilic interaction (HILIC) that might take advantage of the polarity of the analytes. The presence of the $\mathrm{O}$ atom as a substituent on the benzyl group might have improved the HILIC effects of the column hence the observed behaviours of PSI, HO and DMPE compared to the other RP columns. The mobile phase was buffer (100 mM formate) and $\mathrm{MeCN}$ in a ratio 87:13 \% (v/v) at

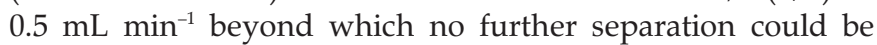
achieved.

\subsection{Ascentis RP-Amide Column}

Compared to alkyl columns, better retention and selectivity for RP-amide was achieved at Buffer:MeCN 35:65 \% (v/v), 100 mM formate. This was expected because, in addition to the hydrophobic interactions, the RP-amide has the H-bond accepting amide carbonyl group that interacted with the $-\mathrm{OH}$ and uncharged $-\mathrm{NH}$ groups of the analytes. The presence of two $\mathrm{H}$-bond donating groups in PSI might have been responsible for its position in the elution order while $\mathrm{HO}$ only has - $\mathrm{OH}$ group. Such groups are absent in PEA implying that its retention was based on the hydrophobic alkyl group while the nucleofugic methoxy group in DMPE could have acted as an H-bond accepting group. However, the $\mathrm{sp}^{2}$-hybridized $\mathrm{O}$ atom of the isoxazole in MUS cannot be an H-bond acceptor. The amide column was the only RP mode that retained MUS and the elution order was different from the phenyl columns. The observed reduction in selectivity of the RP-amide compared to phenyl columns was attributed to the charged state of these basic analytes. Retention was low and separation less achieved at low organic solvent content of the mobile phase.

\subsection{Ascentis HILIC Column}

All the model analytes were separated when the analytes were dissolved in THF and eluted at $0.4 \mathrm{~mL} \mathrm{~min}^{-1}$ with $\mathrm{MeCN}$ :Buffer 90:10 \% (v/v) 10 mM formate. Fig. 3 compares separation when $\mathrm{THF}$ and MeCN diluents were used. Retention, and hence separation, was minimal when analytes were diluted with either $\mathrm{H}_{2} \mathrm{O}$ or $\mathrm{MeOH}$. A general reversal in the elution order compared to

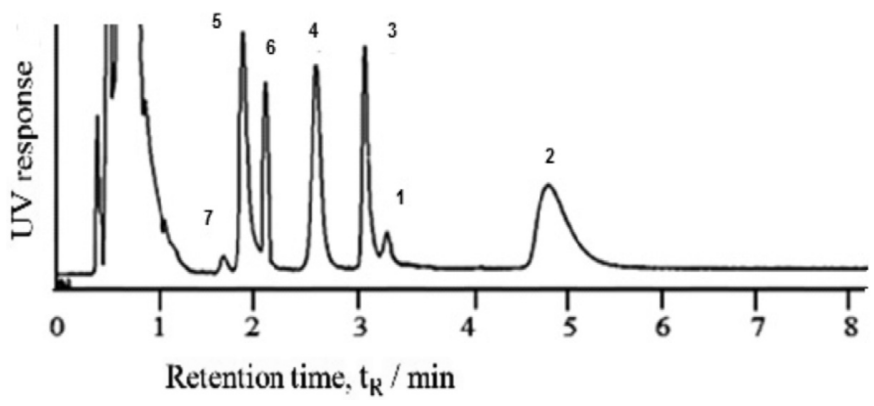

Figure 3 Chromatograms when THF and MeCN were used as diluents for analyte injection and separation achieved on a HILIC column. Compounds: (7) PSI; (6) HO; (5) TA; (4) PEA; (3) DMPE; (2) TRP; (1) MUS

that of the RP approach was observed. In HILIC, a relatively hydrophobic high organic content mobile phase is used to elute analytes through a hydrophilic column. ${ }^{42,46,47,50,53-57}$ The mechanism is the extent of partitioning of analytes between a pseudostagnant aqua layer formed at the surface of the stationary phase and the mobile phase, as confirmed by McCalley and Neue, and Dinh et al. ${ }^{58,59}$ The result is that retention favours the more hydrophilic analytes as confirmed by the negative slope of Fig. 4 .

\subsubsection{The HILIC Complexity}

Our results confirmed that if analyte class is ignored, the general principles of HILIC separation cannot explain the behaviour of some analytes as shown by $\log \mathrm{k} v s \mathrm{X} \log \mathrm{P} 3$ in Fig. 4. This has also been observed by other researchers. ${ }^{42,46}$ This is due to presence of secondary interactions that come into effect based on the structural composition and sterical orientation of the analyte, and the salt concentration of the mobile phase. Such polar basic interactions have been identified as electrostatic, ion exchange, $\mathrm{H}$-bonding and some weak hydrophobic interactions depending on stationary phase, mobile phase and analyte conditions. ${ }^{42,44,46,50,60}$

For analytes where the backbone is similar, the electrostatic effect seemed to be more important as observed with each of the two alkaloid types. The presence of alkyl substituents on the basic $\mathrm{N}$ resulted in a reduction in retention times, implying that the basic $\mathrm{N}$ was involved in these electrostatic interactions. Alkylation was deemed responsible for neutralizing the positive charge on the $\mathrm{N}$ atom ${ }^{55}$. This also allowed for elution order to follow protocol. For example, if phenethylamine or tryptamine derivatives only were compared in Fig. $4, \mathrm{R}^{2}$ values were 0.9882 and 0.9938 , respectively. The resonance-stabilized negative

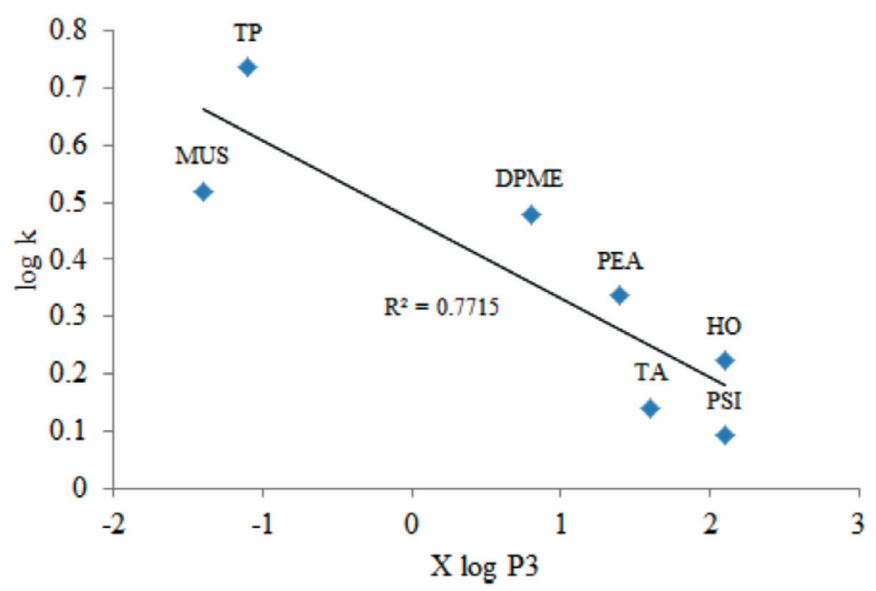

Figure 4 The correlation between polarity and retention as given by log $\mathrm{k}$ vs $\mathrm{X} \log \mathrm{P} 3$. The $\mathrm{k}$ term is the retention factor for each analyte. 
charge on the carboxylic group of TRP enhanced the electrostatic interaction in addition to hydrophilic partitioning hence its observed behaviour together with peak broadening. General peak broadening for those compounds that retain more, as shown by the peak for TRP, has been reported before. ${ }^{42,59}$

\subsubsection{The Moderately Polar Aprotic Diluent for Analyte Injection}

While the emphasis is on using a non-polar aprotic solvent for a mobile phase, solvents that are predicted to give better results than $\mathrm{MeCN}$ are not recommended because of background noise and detection issues proving the irreplaceability of MeCN. ${ }^{45,47,56,60-63}$ Studies by $\mathrm{Li}$ and Huang ${ }^{62}$ showed better separation when $\mathrm{MeCN}$ was used as the mobile phase compared to $\mathrm{MeOH}$, THF and isopropanol. Attempts by Dinh et al. ${ }^{58}$ to admixture THF with the MeCN-containing mobile phase reduced the extent of formation of the water enriched layer on the stationary phase. Also, acetone and THF have very high UV-vis absorbance cut-off wavelengths and show reduced signal response in MS detection. While the recommendations guided by the viscous fingering phenomenon are that the injection solvent must be as close to the mobile phase organic composition as possible with a minimal $50 \% \mathrm{MeCN}$ composition, such declarations have been based on comparison of $\mathrm{MeCN}$ with polar protic solutions like $\mathrm{H}_{2} \mathrm{O}$ and $\mathrm{MeOH} .{ }^{56,60,64}$ It was noted that most publications follow this protocol and rarely report or optimize their injection diluents. Controversies arise considering that the polar protic $\mathrm{MeOH}$ and $\mathrm{H}_{2} \mathrm{O}$ are the solvents of choice for the extraction of polar compounds from biological matrices. This prompted us to try diluting the polar-dissolved stock solutions with the low elution order aprotic solvents. Our research has successfully identified the introduction of a moderately polar aprotic dilution solvent like THF for analyte injection as an essential parameter in $\mathrm{MeCN}$-mobile phase core column HILIC interactions. Compared to $\mathrm{MeCN}$ as an injection eluent at the same ratio, better retention and hence separation was observed when THF was used (Fig. 3). While observations by Dinh et al..$^{58}$ might come into effect, THF is aprotic and relatively apolar and if introduced as an analyte solvent will maximize analyte partitioning into the pseudostagnant water-enriched layer thus counteracting the admixture effect. It was suggested that THF allows for analyte on-column focussing where the analytes are concentrated or accumulated into the pseudo water layer within a narrow range at the column entrance, so that by the time $\mathrm{MeCN}$ mobile phase enters the column the analytes are already retained in the stationary phase. This postulation is based on the review by Jandera et al. ${ }^{63}$ who attributed this on-column focussing to weak eluents. Related to this observation is the use of dichloromethane in place of water for injection of acrylamide by Backe et al ${ }^{65}$ While both dichloromethane and THF have similar polarity (polarity indices of 3.1 and 4 respectively), THF has the advantage of $100 \%$ solubility in water. This implies that THF has the ability to penetrate the pseudo-stagnant aqua layer on the surface of the HILIC column thereby enhancing the partitioning of the analytes into the aqua layer. In HILIC columns where H-bond interactions are less important, THF is a weaker eluent than MeCN based on polarity. The situation is reversed for silica modified columns where $\mathrm{H}$-bond accepting abilities come into effect ${ }^{56,64}$ Peaks were unidentified when analytes were diluted with acetone. Acetone's absorbance cut-off point is $330 \mathrm{~nm}$.

The effect of electrostatic interactions by increasing concentration of formate buffer beyond $10 \mathrm{mM}$ on the extent of separation and retention was inversely minimal and relatively absent for TRP, but seemed to improve sharpness and reduce tailing experienced especially with TRP. Increasing salt concentration is expected to act by competing for electrostatic interaction with the stationary phase hence reducing the effects of electrostatic attractions on retention. TRP also contains a negative charge on its acidic group that tends to cancel out this impact, hence its observed elution status. Other studies where the effect has been more pronounced have identified the residual silanols as the major contributors, a scenario typical of non-endcapped columns. Using a high organic content mobile phase, the embedded water layer on the stationary phase increases, thus reducing amount of available residual silanols and their ionizability. ${ }^{44,50,66,67}$ Also, the low metal impurity implies that potential acidity of the uncapped residual silanols is greatly reduced. ${ }^{46,50}$

\section{Conclusions}

For the analysis of hallucinogens and any other set of polar compounds that contain a basic alkyl-N and aromatic substituents, the phenyl and HILIC-based columns can be considered in order to maximize the number of analytes for simultaneous quantification. Phenyl RP columns are promising through $\pi-\pi$ interactions and could be a first choice for separation depending on the number of compounds, presence of cyclic groups with conjugated $\tau$-electron systems, the class and functional groups of the compounds to be analyzed. Based on studies by other researchers, the pentafluorophenylpropyl stationary phase is also recommended..$^{34,35,37}$ The HILIC is a promising and almost definite choice for separation of polar basic compounds that show separation problems under reverse based phases. The declaration on HILIC separation was based on testing basic alkyl-N hallucinogenic compounds on a fused core silica column. For compounds that are derivatives of a single precursor and where the basic $\mathrm{N}$ has either $\mathrm{H}$ or alkyl substituents, polarity as given by $\mathrm{X} \log \mathrm{P} 3$ values can be used to predict the elution order. The presence of other functional groups attached to this $\mathrm{N}$ atom, as exemplified by the amino acid tryptophan in this study, brings in secondary interactions. Such interactions lead to the collapse of the polarity-based elution predictions. This effect is also observed when other polar biomolecules of a different class or precursor are considered.

Compared to effect of salt concentration, the amount of salt in the mobile phase was more important in fused core silica HILIC retention and the opposite was true for resolution. The reverse was observed for reversed phase chromatography. Our results have shown that in addition to the major parameters deemed essential for HILIC elution optimizations, the injection diluent for analytes also needs to be considered when dealing with polar compounds. The use of relatively non-polar diluents for injection of analytes into a polar mobile phase in HILIC separations was suggested in this research. THF was recommended as the first choice of consideration where acetonitrile or methanol is used as the organic component of the mobile phase.

\section{Acknowledgements}

This study was funded by the Innovation Scholarships programme under the National Research Fund of South Africa (Grant number 94860) and the University of the Witwatersrand postgraduate merit award.

\section{References}

1 D.E. Nichols. Hallucinogens, Pharmacol. Ther., 2004, 101, 131-181.

2 F. Hasler, U. Grimberg, M.A. Benz, T. Huber and F.X. Vollenweider, Acute psychological and physiological effects of psilocybin in healthy humans: a double-blind, placebo-controlled dose-effect study, Psychopharmacology (Berl.), 2004, 172, 145-156.

3 J. Van Amsterdam, A. Opperhuizen and W. Van Den Brink, Harm 
potential of magic mushroom use: a review, Regul. Toxicol. Pharmacol., 2011, 59, 423-429.

4 K. Tsujikawa, K. Kuwayama, H. Miyaguchi, T. Kanamori, Y. Iwata, H. Inoue, et al., Determination of muscimol and ibotenic acid in Amanita mushrooms by high-performance liquid chromatography and liquid chromatography-tandem mass spectrometry, J. Chromatogr. B Anal. Technol. Biomed. Life Sci., 2007, 852, 430-435.

5 R.S. Gable, Comparison of acute lethal toxicity of commonly abused psychoactive substances, Addiction, 2004, 99, 686-696.

6 T. Passie, J. Seifert, U. Schneider and H.M. Emrich, The pharmacology of psilocybin, Addict. Biol., 2002, 7, 357-364.

7 G. Sticht and H. Käferstein, Detection of psilocin in body fluids, Forensic Sci. Int., 2000, 113, 403-407.

8 J.T. Berge, Breakdown or breakthrough? A history of european research into drugs and creativity, J. Creat. Behav., 1999, 33, 257-276.

9 A. Becker, G. Grecksch, H.-G. Bernstein, V. Höllt and B. Bogerts, Social behaviour in rats lesioned with ibotenic acid in the hippocampus: quantitative and qualitative analysis, Psychopharmacology (Berl.), 1999, 144, 333-338.

10 D. Michelot and L.M. Melendez-Howell, Amanita muscaria: chemistry, biology, toxicology, and ethnomycology, Mycol. Res., 2003, $107,131-146$

11 M. Wittmann, O. Carter, F. Hasler, B.R. Cahn, U. Grimberg, P. Spring et al., Effects of psilocybin on time perception and temporal control of behaviour in humans, J. Psychopharmacol., 2007, 21, 50-64.

12 M. Gennaro, D. Giacosa, E. Gioannini and S. Angelino, Hallucinogenic species in Amanita muscaria. Determination of muscimol and ibotenic acid by ion-interaction HPLC, J. Liq. Chromatog. Relat. Technol., 1997, 20, 413-424.

13 F. Hasler, D. Bourquin, R. Brenneisen, T. Bär and F. Vollenweider, Determination of psilocin and 4-hydroxyindole-3-acetic acid in plasma by HPLC-ECD and pharmacokinetic profiles of oral and intravenous psilocybin in man, Pharm. Acta Helv., 1997, 72, 175-184.

14 K. Saito, T. Toyo' oka, M. Kato, T. Fukushima, O. Shirota and Y. Goda, Determination of psilocybin in hallucinogenic mushrooms by reversed-phase liquid chromatography with fluorescence detection, Talanta, 2005, 66, 562-8.

15 J. Chen, M. Li, X. Yan, E. Wu, H. Zhu, K.J. Lee, et al., Determining the pharmacokinetics of psilocin in rat plasma using ultra-performance liquid chromatography coupled with a photodiode array detector after orally administering an extract of Gymnopilus spectabilis, J. Chromatogr. B Anal. Technol. Biomed. Life Sci., 2011, 879, 2669-2672.

16 F.C. Stoermer, K. Janak and G.E. Koller, Ibotenic acid in Amanita muscaria spores and caps, Mycologist, 2004, 18, 114-117.

17 F. Hasler, D. Bourquin, R. Brenneisen and F.X. Vollenweider, Renal excretion profiles of psilocin following oral administration of psilocybin: a controlled study in man, J. Pharm. Biomed. Anal., 2002, 30, 331-339.

18 T. Kamata, M. Katagi and H. Tsuchihashi, Metabolism and toxicological analyses of hallucinogenic tryptamine analogues being abused in Japan, Forensic Toxicol., 2010, 28, 1-8.

19 R. Kysilka, Determination of psilocin in rat urine by high-performance liquid chromatography with electrochemical detection, J. Chromatogr. B Biomed. Sci. Appl., 1990, 534, 287-290.

20 H. Lindenblatt, E. Krämer, P. Holzmann-Erens, E. GouzoulisMayfrank and K.A. Kovar, Quantitation of psilocin in human plasma by high-performance liquid chromatography and electrochemical detection: comparison of liquid-liquid extraction with automated on-line solid-phase extraction, J. Chromatogr. B Biomed. Sci. Appl.,1998, $709,255-263$

21 N. Manevski, M. Kurkela, C. Höglund, T. Mauriala, J. Yli-Kauhaluoma, et al., Glucuronidation of psilocin and 4-hydroxyindole by the human UDP-glucuronosyltransferases, Drug Metab. Dispos., 2010, 38, 386-395.

22 K. Tsujikawa, T. Kanamori, Y. Iwata, Y. Ohmae, R. Sugita, H. Inoue, et al., Morphological and chemical analysis of magic mushrooms in Japan, Forensic Sci. Int., 2003, 138, 85-90.

23 M. Wurst, R. Kysilka and T. Koza, Analysis and isolation of indole alkaloids of fungi by high-performance liquid chromatography, J. Chromatogr. A, 1992, 593, 201-208.

24 J. Bigwood and M. W. Beug, Variation of psilocybin and psilocin levels with repeated flushes (harvests) of mature sporocarps of Psilocybe cubensis (Earle) Singer, J. Ethnopharmacol., 1982, 5, 287-291.
$25 \mathrm{~K}$. Björnstad, O. Beck and A. Helander, A multi-component LC-MS/MS method for detection of ten plant-derived psychoactive substances in urine, J. Chromatogr. B., 2009, 877, 1162-1168.

26 S.D. Brandt and C.P. Martins, Analytical methods for psychoactive N, $\mathrm{N}$-dialkylated tryptamines, TrAC-Trends Anal. Chem., 2010, 29, 858-869.

27 T. Kamata, M. Nishikawa, M. Katagi and H. Tsuchihashi, Optimized glucuronide hydrolysis for the detection of psilocin in human urine samples, J. Chromatogr. B Anal. Technol. Biomed. Life Sci., 2003, 796, 421-427.

28 V. Marcano, A.M. Méndez, F. Castellano, F. Salazar and L. Martinez, Occurrence of psilocybin and psilocin in Psilocybe pseudobullacea (Petch) Pegler from the Venezuelan Andes, J. Ethnopharmacol., 1994, 43, 157-159.

29 R. Martin, J. Schürenkamp, H. Pfeiffer, M. Lehr and H. Köhler, Synthesis, hydrolysis and stability of psilocin glucuronide, Forensic Sci. Int., 2014, 237, 1-6.

30 S. Pichini, E. Marchei, O. García-Algar, A. Gomez, R. Di Giovannandrea and R. Pacifici, Ultra-high-pressure liquid chromatography tandem mass spectrometry determination of hallucinogenic drugs in hair of psychedelic plants and mushrooms consumers, J. Pharm. Biomed. Anal., 2014, 100, 284-289.

31 M. Knox, W. Clark and S. Link, Guantitative analysis of $\beta$-phenethylamines in two Mammillaria species (Cactaceae), J. Chromatogr. A, 1983, 265, 357-362.

32 C. Di Lorenzo, A. Dos Santos, F. Colombo, E. Moro, M. Dell'Agli and P. Restani, Development and validation of HPLC method to measure active amines in plant food supplements containing Citrus aurantium L., Food Control., 2014, 46, 136-142.

33 S. Pichini, L. Morini, R. Pacifici, J. Tuyay, W. Rodrigues, R. Solimini, et al., Development of a new immunoassay for the detection of ethyl glucuronide $(E t G)$ in meconium: validation with authentic specimens analyzed using LC-MS/MS. Preliminary results, Clin. Chem. Lab. Med., 2014, 52, 1179-1185.

34 R.S. Pawar, E. Grundel, A.R. Fardin-Kia and J.I. Rader, Determination of selected biogenic amines in Acacia rigidula plant materials and dietary supplements using LC-MS/MS methods, J. Pharm. Biomed. Anal., 2014, 88, 457-466.

35 F. Pellati and S. Benvenuti, Chromatographic and electrophoretic methods for the analysis of phenetylamine alkaloids in Citrus aurantium, J. Chromatogr. A., 2007, 1161, 71-88.

36 H. Wang, E. J. Walaszczyk, K. Li, Y.-W. Chung-Davidson and W. Li, High-performance liquid chromatography with fluorescence detection and ultra-performance liquid chromatography with electrospray tandem mass spectrometry method for the determination of indoleamine neurotransmitters and their metabolites in sea lamprey, Anal. Chim. Acta, 2012, 721, 147-153.

37 B.C. Nelson, K. Putzbach, K.E. Sharpless and L.C. Sander, Mass spectrometric determination of the predominant adrenergic protoalkaloids in bitter orange (Citrus aurantium), J. Agric. Food Chem., 2007, 55, 9769-9775.

38 M.A. Geyer and F.X. Vollenweider, Serotonin research: contributions to understanding psychoses, Trends Pharmacol. Sci., 2008, 29, 445-453.

39 A.L. Halberstadt, Recent advances in the neuropsychopharmacology of serotonergic hallucinogens, Behav. Brain Res., 2015, 277, 99-120.

40 N. Anastos, N. Barnett, F. Pfeffer and S. Lewis, Investigation into the temporal stability of aqueous standard solutions of psilocin and psilocybin using high performance liquid chromatography, Sci. Justice, 2006, 46, 91-96.

41 T. Cheng, Y. Zhao, X. Li, F. Lin, Y. Xu, X. Zhang, et al., Computation of octanol-water partition coefficients by guiding an additive model with knowledge, J. Chem. Inf. Model., 2007, 47, 2140-2148.

42 R.I. Chirita, C. West, A.L. Finaru and C. Elfakir, Approach to hydrophilic interaction chromatography column selection: application to neurotransmitters analysis, J. Chromatogr. A, 2010, 1217, 3091-3104.

43 D.V. McCalley, Study of the selectivity, retention mechanisms and performance of alternative silica-based stationary phases for separation of ionised solutes in hydrophilic interaction chromatography, J. Chromatogr. A, 2010, 1217, 3408-3417.

44 D.V. McCalley, Is hydrophilic interaction chromatography with silica columns a viable alternative to reversed-phase liquid chromatography for the analysis of ionisable compounds?, J. Chromatogr. A, 2007, 1171, 46-55.

45 M.R. Gama, R.G. da Costa Silva, C.H. Collins and C.B. Bottoli, Hydro- 
philic interaction chromatography, TrAC - Trends Anal. Chem., 2012, $37,48-60$.

46 N.P. Dinh, T. Jonsson and K. Irgum, Probing the interaction mode in hydrophilic interaction chromatography, J. Chromatogr. A, 2011, 1218, 5880-5891.

47 D. García-Gómez, E. Rodríguez-Gonzalo and R. Carabias-Martínez, Stationary phases for separation of nucleosides and nucleotides by hydrophilic interaction liquid chromatography, $\operatorname{Tr} A C$ - Trends Anal. Chem., 2013, 47, 111-128.

48 Y. Kawachi, T. Ikegami, H. Takubo, Y. Ikegami, M. Miyamoto and N. Tanaka, Chromatographic characterization of hydrophilic interaction liquid chromatography stationary phases: hydrophilicity, charge effects, structural selectivity, and separation efficiency, J. Chromatogr. A, 2011, 1218, 5903-5919.

49 Y. Guo and S. Gaiki, Retention and selectivity of stationary phases for hydrophilic interaction chromatography, J. Chromatogr. A, 2011, 1218, 5920-5938.

50 B. Buszewski and S. Noga, Hydrophilic interaction liquid chromatography (HILIC) - a powerful separation technique, Anal. Bioanal. Chem., 2012, 402, 231-247.

51 A. Kumar, J.C. Heaton and D.V. McCalley, Practical investigation of the factors that affect the selectivity in hydrophilic interaction chromatography, J. Chromatogr. A, 2013, 1276, 33-46.

52 E. Loeser and P. Drumm, Using strong injection solvents with $100 \%$ aqueous mobile phase in RP-LC, J. Sep. Sci., 2006, 29, 2847-2852.

53 P. Hemström and K. Irgum, Hydrophilic interaction chromatography, J. Sep. Sci., 2006, 29,1784-1821.

54 A.J. Alpert, Hydrophilic-interaction chromatography for the separation of peptides, nucleic acids and other polar compounds, J. Chromatogr. A, 1990, 499, 177-196.

55 P.J. Boersema, S. Mohammed and A.J. Heck, Hydrophilic interaction liquid chromatography (HILIC) in proteomics, Anal. Bioanal. Chem., 2008, 391, 151-159.

56 A.E. Karatapanis, Y.C. Fiamegos and C.D. Stalikas, A revisit to the retention mechanism of hydrophilic interaction liquid chromatography using model organic compounds, J. Chromatogr. A, 2011, 1218, 2871-2879.
57 B. Dejaegher and Y. Vander Heyden, HILIC methods in pharmaceutical analysis, J. Sep. Sci. 2010, 33, 698-715.

58 N.P. Dinh, T. Jonsson and K. Irgum, Water uptake on polar stationary phases under conditions for hydrophilic interaction chromatography and its relation to solute retention, J. Chromatogr. A, 2013, 1320, 33-47.

59 D.V. McCalley and U.D. Neue, Estimation of the extent of the water-rich layer associated with the silica surface in hydrophilic interaction chromatography, J. Chromatogr. A, 2008, 1192, 225-229.

60 B. Chauve, D. Guillarme, P. Cléon and J. Veuthey, Evaluation of various HILIC materials for the fast separation of polar compounds, J. Sep. Sci., 2010, 33, 752-764

61 K.J. Fountain, J. Xu, D.M. Diehl and D. Morrison, Influence of stationary phase chemistry and mobile-phase composition on retention, selectivity, and MS response in hydrophilic interaction chromatography, J. Sep. Sci., 2010, 33, 740-751.

62 R. Li and J. Huang, Chromatographic behavior of epirubicin and its analogues on high-purity silica in hydrophilic interaction chromatography, J. Chromatogr. A, 2004, 1041, 163-169.

63 P. Jandera, Stationary and mobile phases in hydrophilic interaction chromatography: a review, Anal. Chim. Acta, 2011, 692, 1-25.

64 J. Ruta, S. Rudaz, D.V. McCalley, J.L. Veuthey and D. Guillarme, A systematic investigation of the effect of sample diluent on peak shape in hydrophilic interaction liquid chromatography, J. Chromatogr. A, 2010, 1217, 8230-8240.

65 W.J. Backe, V. Yingling and T. Johnson, The determination of acrylamide in environmental and drinking waters by large-volume injection - hydrophilic-interaction liquid chromatography and tandem mass spectrometry, J. Chromatogr. A, 2014, 1334, 72-78.

66 S. Noga, S. Bocian and B. Buszewski, Hydrophilic interaction liquid chromatography columns classification by effect of solvation and chemometric methods, J. Chromatogr. A, 2013, 1278, 89-97.

67 F. Gritti, A. dos Santos Pereira, P. Sandra and G. Guiochon, Comparison of the adsorption mechanisms of pyridine in hydrophilic interaction chromatography and in reversed-phase aqueous liquid chromatography, J. Chromatogr. A, 2009, 1216, 8496-8504. 